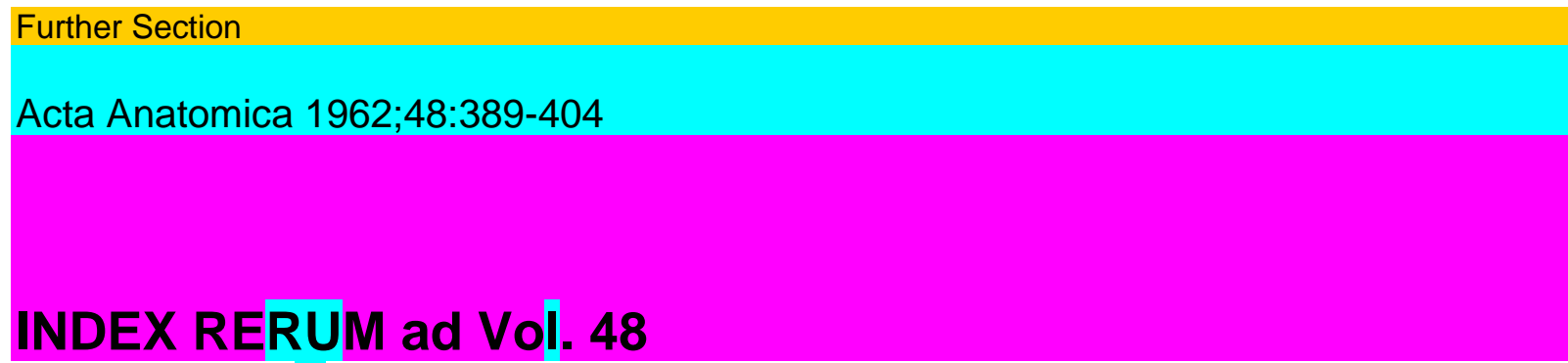

Confecit G. Boehm, Basel

Abortive Mitosen (C) 173 (185)

Acalcemic conditions, v. Acellular bone

Acellular bone of teleost fish, studies (II.: Response to fracture under normal

and acalcemic conditions) 46

Acide desoxyribonucléique, v. ADN

Acides amines, marques en S35, C14 et H3, v. Milz

Adipeux, tissu adipeux; genèse et structure du tissu adipeux chez Thomme 232

-; la lipogenèse au cours de la formation du tissu adipeux 316

Adipogenèse, v. Adipeux . . .

Adipose tissue, v. Adipeux ...

ADN (= acide desoxyribonucléique); teneur en ADN et volume du noyau (C) 179

(ADN; variations du volume nucléaire et de la teneur en $\mathrm{ADN}$ du noyau en

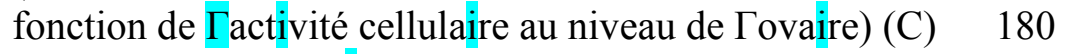

Adrenals, v. Nebenniere

Adultes, v. Tissu adipeux

Age-groups; the external elastic layer of the cerebral arteries in different

age-groups

Air-passages of the dugong lung

95

Alter, v. Age-groups

Aminosäuren, radioaktiv mit S35, C14 und H3 markierte, v. Milz

Amitose; neuere Arbeiten fiber die Amitose (C) 174

-; autorenouvellement des cellules du lobe antérieur hypophysaire par ami

tose. Role de Гendocytogénèse $(\mathrm{C}) 177$ (185)

- $\quad$ nach partieller Hepatektomie, cytophotometrische Befunde (C) .... 178

-; introduction au problème de Гamitose (C) 173

-; Schlußbemerkungen zum Problem der Amitose (C)

181

- und Zellfunktion, ihr Verhältnis (C) 175

-; v. Divisions nucléaires

Amitoses, classification (C)

174

Amitotische Kernteilung und Endocytogenese (C)

Anas, v. Pancreas

Anatomenkongreß, europäischer, zweiter (1963)

Anatomenkongreß, internationaler, achter (1965)

Anatomie; Atlas der systematischen Anatomie des Menschen (Bd. 1) (2.Aufl.)

(B) 189

-; v. Leonard de Vinci (B)

Anatomische Gehirnsektion (B) 
$1(B)=$ New books - Livres nouveaux - Buchbesprechungen $(C)=$ Report or demonstration Communication ou demonstration - Vortrag oder Demonstration

390

Index rerum

Anatomy; regional and applied (second ed.) (B)

Angiography, v. Epiphysis

Anoxémie, v. Juxtaglomérulaire

Antidiuretisches Hormon, Angriffspunkt am Nephron (C) 186

Antimitotiques, substances; le mécanisme normal de la mitose et son analyse

par Faction des substances antimitotiques (C) 168

-; v. (Colchicin)

Appareil juxtaglomérulaire, investigations 368

Applied and regional anatomy (second ed.) (B) 294

Arteries, cerebral; the external elastic layer of the cerebral arteries in dif

ferent age-groups

1

Arteries, extracranial cerebral, v. Cerebral infarction (B)

Articulation du coude, v. Elbow joint

Articulation de la hanche, v. Hip joint

Articulatio coxae, v. Hip joint

Articulatio cubiti, v. Elbow joint

Atlas der systematischen Anatomie des Menschen (Bd. 1) (2.Aufl.) (B) . 189

Auge, v. Sclera

Auris, v. Gehörorgan (B)

Autoradiographische Untersuchungen des Eiweiß-Stoffwechsels der Säuger-

milz nach Gabe von S35-, C14- und H;î-markierten Aminosäuren ... 7

Autorenouvellement, v. Hypophysis

Aves, v. Sclera

Basalganglien (Primatologia Bd. 2, Teil 2, Lief. 8) (B) 191

Bernsteinsäure-dehydrogenase, v. Succinic dehydrogenase Beutelratte, v. Corpus striatum

Beuteltiere, v. Corpus striatum

Biological stains (7th ed.) (B) 188

Bone, acellular, of teleost fish (II.: Response to fracture under normal and

acalcemic conditions)

46

-; v. Human epiphysis

Bourgeons du gout, v. Succinic dehydrogenase

Bourgeons musculaires cultives in vitro, contribution à $\Gamma$ étude du problème

de la multiplication nucléaire (C) 178

Brain, v. Basalganglien (B), Cerebral arteries, Cerebral infarction (B), Corpus

striatum, Gehirn (B), Gehirnsektion (B), Lemniscus

Buchbesprechungen $187 \mathrm{ff}$, $293 \mathrm{ff}$.

Bullfrog, v. Pancreas

C14, v. Milz

Calcium (Acalcemic conditions), v. Acellular bone

Caliculi gustatorii, v. Succinic dehydrogenase

Index rerum 391

Canard, v. Pancreas 
Capra, v. Goat

Carassius auratus, v. Teleost fish

Carbon, monoxyde de carbone, v. Juxtaglomérulaire

Carbon, radioactive, v. Milz

Caryocinèse; sur une théorie physico-chimique et colloïdale de la caryo-

cinèse $(\mathrm{C}) \quad 171$

Caryométrie, v. Kerngrößenunterschiede, Zellkernvolumen

Cavum nasi, v. Rats, germfree- ...

Cellular bone, v. Acellular bone

Cell..., v. Zell...

Cerebral arteries; the external elastic layer of the cerebral arteries in dif

ferent age-groups

Cerebral infarction: The role of stenosis of the extracranial cerebral arteries

(B) 189

Cerebrum, v. Basalganglien (B), Corpus striatum, Gehirn (B), Gehirnsektion (B), Lemniscus

Cerveau, v. Cerebrum

Chèvre, v. Lemniscus

Chicken, v. Gonades embryonnaires, Parabiose hétérogène, Sclera

Children, v. Tissu adipeux

Chlorure de soude, v. Nebenniere

Chorioidea; the development of the sclera and choroid in staged chick em

bryos 335

Choroid, v. Chorioidea

(Chromatoïdes; les formations chromatoïdes dans les mitoses de segmenta

tion $(\mathrm{C}) \quad 185)$

Chromosomes; intérêt des cultures de moelle osseuse pour Гétude des chro mosomes humains (C) 169

Classification des amitoses (C)

$\mathrm{CO}$, v. Juxtaglomérulaire

(Colchicin, v. Karyometrische Untersuchungen)

-; v. Antimitotiques ...

Colloïdale, théorie colloïdale, v. Caryocinèse

Coloration, v. Stains (B), Vestopal-W

Comparative anatomy, v. Corpus striatum

Comptes rendus du IV. Symposium histologicum internationale (1961) (C) $168 \mathrm{ff}$.

Congrès Fédératif International d'Anatomie, VIПe (1965) 192

Contractions d'explants myocardiques, dispositif photoélectrique pour en-

registrer des courbes $(\mathrm{C}) \quad 185$

Corpus pineale, v. Pineal

Corpus striatum; zur feineren Struktur und vergleichenden Anatomie des

Corpus striatum primitiver Beutel- und Nagetiere 347

392 Index rerum

Coude, articulation de la coude, v. Elbow joint

Croissance et les divisions nucléaires, en particulier l'amitose (C) .... 168 if.

Croissance, v. Human epiphysis

Cryostat, v. Phosphatase 
Culture de tissus, v. Bourgeons musculaires, Gonades embryonnaires, Mitochondries, Myocardiques, Parabiose hétérogène, Souris, Zellteilung Culture de moelle osseuse, intérêt pour Гétude des chromosomes humains (C) 169

Cultures de fibroblastes, lesions et mitoses (C) 172

Cytochemistry, v. Hip joint, Histochemie(B), Phosphatase, Succinic dehydrogenase, Transitional epithelium Cytophotometrische Befunde zur Amitose nach partieller Hepatektomie (C) 178

Dentes, v. Zähne

Déshydrogénase succinique, v. Succinic dehydrogenase

258335

$168 \mathrm{ff}$.

95

Desoxyribonucleic acid, v. ADN

Development of the human hip joint, histochemical studies ...

Development of the sclera and the choroid in staged chick embryos

Development, v. Tissu adipeux, Zähne

Développement de la souris, v. Souris

de Vinci, Leonard, v. Leonard de Vinci

Didelphís marsupíalís, v. Corpus striatum

Dispositif photoélectrique, v. Contractions d'explants myocardiques

Diurese (antidiuretisches Hormon), v. Nephron

Divisions nucléaires et croissance, en particulier l'amitose (C) . .

Duck, v. Pancreas

Dugong lung, air-passages

Ear, v. Gehörorgan (B), Primatologia (B)

Eiweiß-Stoffwechsel der Säugermilz nach Gabe von S35-, C14- und H3-mar-

kierten Aminosäuren, autoradiographische Untersuchungen .... 7

Elastic layer, external, of the cerebral arteries in different age-groups . . 1

Elbow joint; stabilization of and load on the elbow joint in some protective

movements (An experimental study) 224

Electron microscopy, v. Electroniques..., Elektronenmikroskopie

Electroniques, micrographes électroniques du rein (C) $\quad 187$

(-; [Foie, rein, protozoaires etc.] [C] 185)

-; v. Elektronenmikroskopie, Phosphatase, Transitional epithelium Elektronenmikroskopie;

Beitrag der Elektronenmikroskopie zur Kenntnis

der Kern- und Zellteilung (C) 171

- ; v. Electroniques

Ellbogengelenk, v. Elbow joint

Embryo, v. Hip joint, Sclera, Tissu adipeux, Zähne

Embryon de poulet, v. Gonades embryonnaires, Parabiose hétérogène

Index rerum 393

Endocrinology, v. Reprints

Endocytogenese und amitotische Kernteilung (C) 177

Endocytogénèse; autorenouvellement des cellules du lobe antérieur hypo-

physaire par amitose. Role de Гendocytogénèse (C) 177 (185)

Enfants, v. Tissu adipeux 
Ente, v. Pancreas

Epiphysis cerebri, v. Pineal

Epiphysis, human; the vascular pattern of a growing and fullgrown human

epiphysis $\quad 156$

Epithelium, transitional, of the rat urinary bladder in response to induced

physiological changes, histology, histochemistry and electron micros

copy 297

(Epitheloberfläche, interzellulärer Verschluß (C)

Erwachsene, v. Tissu adipeux

European Anatomical Meeting, second (1963)

Explants myocardiques, v. Myocardiques

Extracranial cerebral arteries, v. Cerebral infarction (B)

Eye, v. Sclera

Färbung, v. Stains (B), Vestopal-W

Fascia cruris (Fascia antibrachii), v. Fascien

Fascien, Textur und Vascularisation

61

Fatty tissue, genesis, v. Tissu adipeux

Feinstruktur, v. Electroniques..., Elektronenmikroskopie, Phosphatase,

Transitional epithelium Femoral muscles, posterior; the length and mode of termination of individual

muscle fibres in the human sartorius and posterior femoral muscles . 242 Ferments, v.

Phosphatase, Succinic dehydrogenase Fettgewebe des Menschen, Genese, v. Tissu adipeux

Fetus, v. Foetus

Fibres musculaires individuelles, terminaison, v. Muscle fibres

Fibroblastes; lesions et mitoses dans les cultures de fibroblastes (C) . . 172 Film, v. Mitose Fish,

v. Teleost fish

Foetus, v. Hip joint, Sclera, Tissu adipeux, Zähne Foie en parabiose hétérogène avec d'autres

organes d'embryon de poulet,

comportement en culture (C) 183

Foie, v. Hepatektomie, Leber, Micrographies, Microsomes Fractures of the operculum and of lower jaw elements, v. Teleost fish Frog (bullfrog), v. Pancreas Frosch (Ochsenfrosch), v.

Pancreas

Gallus gallus, v. Gonades embryonnaires, Parabiose hétérogène, Sclera

Acta anat,, Vol. 48, No. 4 (1962) 27

394

Index rerum

Gammastrahlen (Co60), v. Souris

Gefäße, v. Cerebral arteries, Cerebral infarction (B), Epiphysis, Гascien

Gehirn, Präparation (eine Anleitung zum makroskopischen Studium) (B) . 187

-; v. Gehirnsektion (B)

Gehirn, v. Basalganglien (B), Cerebral arteries, Cerebral infarction (B), Corpus striatum,

Gehirnsektion (B), Lemniscus

Gehirnsektion, anatomische (B) 188

Gehörorgan der Wirbeltiere und des Menschen (B) 190

Gelenk, v. Elbow joint

Genomsonderung in den Mitosen dor Rattenleber (C) 
Germfree-reared and ex-germfree rats, histology of the nasal and paranasal cavities $\quad 108$

Gesohlechtsbedingte Kerngrößenunterschiede und ihre statistische Sicherung $(\mathrm{C}) \quad 180$

Geschmacksknospen, v. Succinic dehydrogenase

Gesellschaftsberichte (C) $168 \mathrm{ff}$.

Gewebekultur, v. Bourgeons musculaires, Гibroblastes, Gonades embryonnaires, Mitochondries, Moelle osseuse, Myocardiques, Parabiose hétéro-gène, Souris, Zellteilung

Giraffa camelopardalís, v. Giraffenplacenta

Giraffenplacenta, ein Beitrag zu ihrem Bau 206

Glande de Loewenthal, v. Pineal

Glande pineal, v. Pineal

Glandula parathyreoidea, v. Teleost fish

Glandula suprarenalis, v. Nebenniere

Glykogen, v. Tageszyklische Untersuchungen

Goat; the medial lemniscus in the goat

Goldhamster, v. Corpus striatum

Gonades embryonnaires de poulet, cultivées in vitro, action des rayons X (C) 184

Grenouille-taureau, v. Pancreas

Gros sel, v. Nebenniere

Growing human epiphysis, v. Vascular pattern

Gustatory epithelia, v. Succinic dehydrogenase

H3, v. Milz

Halicore dugong, v. Dugong lung

Hamster, v. Corpus striatum

Hanche, articulation de la hanche, v. Hip joint

Handbuch der Histochemie (Bd. 111, Nucleoproteide: 2.Teil: Ribonuclein-

säure und Proteinsynthese, Ribonucleinsäure und Morphogenese, Lo-

kalisation der Ribonucleinsäure im Vertebratenorganismus, Methoden

des Proteinnachweises) (B) 295

Harnblase der Ratte, v. Transitional epithelium

Index rerum 395

Hepar, v. Foie, Hepatektomie, Leber, Micrographies, Microsomes

Heparin, Wirkung auf die Zellteilung und die Proliferationsprozesse (C) . 185

Hepatektomie; cytophotometrische Befunde zur Amitose nach partieller $\mathrm{He}$

patektomie (C)

178

Hétérogène, $v$. Parabiose hétérogène

Hip joint; histochemical studies of the development of the human hip joint 258

Histochemical studies of the development of the human hip joint .... 258

Histochemie, Handbucb (Bd.III Nucleoproteide: 2.Teil: Ribonucleinsäure

und Proteinsynthese, Ribonucleinsäure und Morphogenese, Lokali-

sation der Ribonucleinsäure im Vertebratenorganismus, Methoden des

Proteinnachweises) (B) 295

Histochemistry, v. Phosphatase, Succinic dehydrogenase, Transitional epithelium Histologie; comptes rendus du IV. Symposium histologicum Internationale (1961) (C) $168 \mathrm{ff}$. 
Histologie des Menschen, Leitfaden (5. Aufl.) (B) 293

Histology, v. Transitional epithelium

Historie, v. Leonard de Vinci

Hormon, antidiuretisches, Angriffspunkt am Nephron (C) 186

Hüftgelenk, v. Hip joint

Hühnchen, v. Gonades embryonnaires, Parabiose hétérogène, Sclera

Human epiphysis, v. Epiphysis

Human hip joint, v. Hip joint

Human sartorius, v. Musculus sartorius

Humerus, v. Epiphysis

(Hydratation du noyau, v. Volume)

Hypophysis; autorenouvellement des cellules du lobe antérieur hypophysaire

par amitose. Role de Гendocytogénèse (C) 177 (185)

Infarction, v. Cerebral infarction (B)

Inhibition; nervous inhibition (Proceedings of the Second Friday Harbor

Symposium) (B) 190

Innervation der menschlichen Zähne, Entwicklung 322

International Anatomical Congress, eight (1965) 192

Ischémie, v. Juxtaglomérulaire

Islets of Langerhans, cellular composition in the bullfrog, Rana catesbíana 149

-; influence of the islet A and B cells on the exocrine pancreatic tissue in

the duck 137

Isotope, radioaktive, v. Milz

Jaw, lower jaw, v. Acellular bone

Joint, v. Elbow joint

Juxtaglomérulaire; appareil juxtaglomérulaire, investigations

368

396

Index rerum

Kalziumfreie Nahrung, v. Acellular bone

Karyokinese, v. Caryocinèse

Karyometrie, v. Kerngrößenunterschiede, Zellkernvolumen

(Karyometrische Untersuchungen am durch Colchicin beeinflußten Regene-

rationsblastem vom Salamander (C) 180)

Keimfrei ernährte Ratten, v. Rats, germfree-... Kerne, v. Divisions nucléaires

Kernfragmentierung, Kernknospung und Kernlappung (C) 175

Kerngrößenunterschiede, geschlechtsbedingte, und ihre statistische Siche-

rung (C) $\quad 180$

Kernknospung, Kernlappung und Kernfragmentierung (C) 175

Kernlappung, Kernknospung und Kernfragmentierung (C) 175

Kernteilung, amitotische, und Endocytogenese (C) 177

Kernteilung mit und ohne Spindel (C) 169

-; v. Amitose, Elektronenmikroskopie, Mitose Kernvolumen, v. Zellkernvolumen

Kernwachstum, rhythmisches (C) 179

Kidney, v. Electroniques, Juxtaglomérulaire, Micrographies, Nephron,

Phosphatase Kinder, v. Tissu adipeux Kitchen-salt, v. Nebenniere Knochen, v. Human epiphysis 
Knochenbrüohe (Operculum und Unterkieferteile), v. Teleost fish Knochenfische, v. Teleost fish Kochsalzangebot, v. Nebenniere Kohlenoxyd, v. Juxtaglomérulaire Kohlenstoff, radioaktiver, v. Milz

Langerhans, islets, cellular composition in the bullfrog, Rana catesbiana . 149 -; influence of the islet $\mathrm{A}$ and $\mathrm{B}$ cells on the exocrine pancreatic tissue in

the duck 137

Lamantin (Halicore Dugong), v. Dugong lung Langue, v. Succinic dehydrogenase

Leber; Genomsonderung in den Mitosen der Rattenleber (C) $\quad 170$

-; Rattenleber, tageszyklische Untersuchungen (C) 182 (186)

-; v. Foie, Hepatektomie, Micrographies, Microsomes Lehrbuch, v. Atlas (B), Anatomy (B),

Leitfaden (B)

Leitfaden der Histologie des Menschen (5. Aufl.) (B) 293

Lemniscus, medial, in the goat

Leonard de Vinci, dessins anatomiques (B) 293

Lesions et mitoses dans les cellules de fibroblastes (C) 172

Library of the Institute of Experimental Medicine and Surgery of the Uni

versity of Montreal, reconstruction 296

Index rerum 397

Libri 187 ff., $293 \mathrm{ff}$.

Light microscopy, v. Vestopal-W

Lien, v. Milz

Lingua, v. Succinic dehydrogenase

Lipides, formation, v. Tissu adipeux

Lipochondries et mitochondries dans les cellules en culture (film) (C) . . 183

Lipogenèse au cours de la formation du tissu adipeux 316

-; v. Tissu adipeux

Liver, v. Foie, Hepatektomie, Leber, Micrographies, Microsomes

Livres nouveaux $187 \mathrm{ff}$., $293 \mathrm{ff}$.

Loew $\beta$ nthal, glande de Loewenthal, v. Pineal

Lung of dugong, air-passages 95

"Lymphatic system"; some new data concerning the functional unity of the

"lymphatic system" 114

Mâchoire inférieure, v. Acellular bone

Mammalia, v. Dugong lung, Milz

Man, v. Anatomie (B), Chromosomes, Epiphysis, Elbow joint, Gehörorgan

(B), Hip joint, Histologie (B), Muscle fibres, Tissu adipeux, Zähne Mandibula, v. Acellular bone Marsupialia, v. Corpus striatum Mastocytes, v. "Lymphatic system" Maus, v. Souris Maxillaire inférieur, v. Acellular bone

Medial lemniscus in the goat 193

Mesocrícetus auratus, v. Corpus striatum

Metabolismus, v. Eiweiß-Stoffwechsel

Methode, v. Contractions d'explants myocardiques, Gehirn (B), Gehirn-

sektion (B), Histochemie (B), Stains (B), Vestopal-W Mice, v. Souris

Micrographies électroniques (Foie, rein, protozoaires etc.) (C) 185

Micrographies électroniques du rein (C) 187

Microscope électronique, v. Électroniques 
Microscopy, light microscopy, v. Vestopal-W

Microsomes; fractionnement des structures microsomiales du foie. Etude biochimique et morphologique (C) 181

Milz; autoradiographische TJntersuchungen des Eiweiß-Stoffwechsels der Säugermilz nach Gabe von S35-, C14- und H3-markierten Aminosäuren 7 Mitochondries et lipochondries dans les cellules en culture (film) (C) . . 183 Mitose; le mécanisme normal de la mitose et son analyse par Faction des substances antimitotiques $(\mathrm{C})$

-; (analyse par film (C) 168

-; v. Amitose, Divisions nucléaires, Elektronenmikroskopie, Kernteilung 398

Index rerum

Mitosen, abortive (C) 173 (185)

Mitosen der Rattenleber, Genomsonderung (C) 170

Mitoserate und Zellkernvolumen in der Zona glomerulosa und Zona fasciculata der Ratten-Nebenniere bei erhöhtem und erniedrigtem Koch-

salzangebot, Schwankungen (C) $\quad 180$

Mitoses et lesions dans les cultures de fibroblastes (C) 172

(Mitoses de segmentation, les formations chromatoïdes (C) 185)

Moelle osseuse, cultures, intérêt pour Гétude des chromosomes humains (C) 169

Monoxyde de carbon, v. Juxtaglomérulaire

Montreal; library of the Institute of experimental Medicine and Surgery of the University of Montreal, reconstruction 296

Morphodynamie nucléaire, v. Pineal

Mouse, v. Souris

Mouvements dit «de protection* ${ }^{*}$. Elbow joint

Mucosa (Cavum nasi), v. Rats, germfree- ...

Mucosa (urinary bladder), v. Transitional epithelium

Multiplication nucléaire, v. Bourgeons musculaires

Mus musculus, v. Souris

Muscle fibres; the length and mode of termination of individual muscle fibres

in the human sartorius and posterior femoral muscles $\quad 242$

Muscles; contribution à Гétude du problème de la multiplication nucléaire

dans les bourgeons musculaires cultivés in vitro (C) 178

Musculus biceps femoris, v. Muscle fibres

Musculus sartorius; the length and mode of termination of individual muscle

fibres in the human sartorius and posterior femoral muscles .... 242 Musculus semimembranosus,

v. Muscle fibres Musculus semitendinosus, v. Muscle fibres Muskelfasern, Endausbreitung, v.

Muscle fibres Myocardiques, explants -; dispositif photoélectrique pour enregistrer des

courbes de contractions d'explants myocardiques (C)

185

Nachrichten 192, 296

$\mathrm{NaCl}$, v. Nebenniere

Nagetiere, v. Corpus striatum

Nasal and paranasal cavities, histology, of germfree-reared and ex-germfree

rats 108 
Nebenniere; Schwankungen des Zellkernvolumens und der Mitoserate in der Zona glomerulosa und Zona fasciculata der Ratten-Nebenniere bei er höhtem und erniedrigtem Kochsalzangebot (C) 180

Nephron, Angriffspunkt des antidiuretischen Hormons (C) 186

Nerven (Nervensystem, Zentralnervensystem), v. Basalganglien (B), Cerebral arteries, Cerebral infarction (B), Corpus striatum, Lemniscus, Nervous inhibition, Zähne

Index rerum

399

Nervous inhibition (Proceedings of the Second Friday Harbor Symposium)

(B) $\quad 190$

New books 187 ff., 293 ff.

News 192, 296

Niere, v. Electroniques, Juxtaglomérulaire, Micrographies, Nephron, Phosphatase

Nouvelles 192, 296

Noyau; (variations du volume nucléaire et de la teneur en ADN du noyau

en fonction de $\Gamma$ activité cellulaire au niveau de $\Gamma$ ovaire (C) 180)

-; volume, et teneur en $\mathrm{ADN}(\mathrm{C}) \quad 179$

-; (volume, poids sec et hydratation (C) 179)

-; v. Bourgeons musculaires, Divisions nucléaires, Kern...

Nucléaire, multiplication nucléaire, v. Bourgeons musculaires

Nuclei, v. Kern...

Nucleoproteide, v. Histochemie (B)

Ochsenfrosch, v. Pancreas Oculus, v. Sclera Oeil, v. Sclera

Ohr (Primatologia, Bd. 2, 5. Lieferung) (B) 295

Ontogenesis, v. Hip joint, Sclera, Souris, Tissu adipeux, Zähne

Operculum, v. Acellular bone

Oreille, v. Gehörorgan (B), Primatologia (B)

Organes embryonnaires, v. Parabiose hétérogène

Organum gustus, v. Succinic dehydrogenase

Organum stato-acusticum, v. Gehörorgan (B), Ohr (B)

Os, v. Acellular bone, Human epiphysis

Osmium fixation, v. Vestopal-W

Osteocytes, v. Acellular bone

(Ovaire, activité cellulaire, v. ADN)

Oxygen, v. Juxtaglomérulaire

Pancreas; cellular composition of the islets of Langerhans in the bullfrog, Rana catesbíana $\quad 149$

-; influence of the islet A and B cells on the exocrine pancreatic tissue in the duck 137

Parabiose hétérogène; comportement, en culture, du foie en parabiose hé térogène avec d'autres organes d'embryon de poulet (C) 183

Paranasal and nasal cavities, histology, of germfree-reared and ex-germfree rats 108

Phosphatase alcaline non spécifique dans le rein, demonstration à $\Gamma$ aide du cryostat et du microscope électronique (C) 187

400 


\section{Index rerum}

Photoelektrische Einrichtung, zur Registrierung, v. Contractions d'explants

myocardiques Physiological changes, induced in the transitional epithelium, v. Transitional epithelium Pineal; sur $\Gamma$ effet de $\Gamma$ extrait pineal sur la morphodynamique (C) .... 176 Pisces, v. Teleost fish Pituitary gland, v. Hypophysis

Placenta; Beitrag zum Bau der Giraffenplacenta 206

Plasma cells, v. "Lymphatic system"

(Poids sec du noyau, v. Volume)

Poissons, v. Teleost fish

Poulet, v. Gonades embryonnaires, Parabiose hétérogène, Sclera

Poumon, v. Dugong lung

Primatologia (Bd. 2, Teil 2, Lief. 8: Basalganglien) (B) 191

Primatologia (Bd. 2, Lief. 5: Das Ohr) (B) 295

Problème de Гamitose, introduction (C) 173

Proliferationsprozesse; Wirkung des Heparins auf die Zellteilung und die

Proliferationsprozesse (C) 185

Protective movements, v. Elbow joint Proteine (Synthese, Nachweis usw.), v. Histochemie (B)

Proteins, metabolism, v. Eiweiß-Stoffwechsel Protozoaires, v. Micrographies

(Pseudoamitoses par rayons $X$ (avec film) (C) 172)

Pulmo, v. Dugong lung

Radiation, v. Gonades embryonnaires, Pineal, Rayons X, Souris

Radiographie, v. Autoradiographische Untersuchungen...

Rana catesbíana, v. Pancreas

Rate, v. Milz

Rats, germfree-reared and ex-germfree; histology of the nasal and paranasal cavities of germfree-reared and ex-germfree rats 108

-, v. Transitional epithelium

Rattenleber; Genomsonderung in den Mitosen der Rattenleber (C) .... 170

-; tageszyklische Untersuchungen (C) 182 (186)

Rattennebenniere; Schwankungen des Zellkernvolumens und der Mitoserate

in der Zona glomerulosa und Zona fasciculata der Ratten-Nebenniere

bei erhöhtem und erniedrigtem Kochsalzangebot (C) $\quad 180$

Rayons gamma (Co60), v. Souris

Rayons X; action sur les gonades embryonnaires de poulet cultivées in vitro

(C) 184

-; (les pseudoamitoses par rayons X (avec film) (C) 172)

$-;$ v. Pineal

(Regenerationsblastem, v. Karyometrische Untersuchungen)

Index rerum 401

Regional and applied anatomy (second ed.) (B) 294

Rein; investigations sur Гappareil juxtaglomérulaire 368

-; v. Electroniques, Micrographies, Phosphatase, Nephron Ren, v. Electroniques, Micrographies, Nephron, Phosphatase, Rein Reprints for the library of the Institute of Experimental Medicine and Surgery of the University of Montreal (for their reconstruction) .... 296

Reunion Européenne d'Anatomie, deuxième (1963) 192

Rhythmisches Kernwachstum (C) 179 
Rhythmus; tageszyklische Untersuchungen an Rattenlebern (C) . . 182 (186) Ribonucleinsäure (Lokalisation, Proteinsynthese, Morphogenese), v. Histo-

chemie (B) Rodentía, v. Corpus striatum

Röntgenstrahlen, v. Gonades embryonnaires, Pineal, Rayons X Rongeurs, v. Corpus striatum S35, v. Milz

Säugermilz, v. Milz

(Salamander, v. Karyometrische Untersuchungen)

Sauerstoífmangel, v. Juxtaglomérulaire

Schutzbewegungen, v. Elbow joint

Schwefel, radio aktiver, v. Milz

Sclera; the development of the sclera and the choroid in staged chick embryos 335

Seekuh, v. Dugong lung

Septa intermuscularia, v. Fascien

Sex, v. Kerngrößenunterschiede

Sinus craniens, v. Rats, germfree-...

Sitzungsberichte (C) $168 \mathrm{ff}$.

Skeletal calcium, mobilisation, v. Teleost fish

Skeletal muscles, v. Muscle fibres

Sociétés (C) 168 Í

Society transactions (C) $168 \mathrm{ff}$.

Sodium chloride, v. Nebenniere

Soufre, radioactive, v. Milz

Souris; recherches sur le développement de la souris, en cours au laboratoire

d'embryologie du Comité national pour Гénergie nucléaire (C.N.E.N.)

de Frascati (C) 184

Spindel; Kernteilung mit und ohne Spindel (C)

169

Spleen, v. Milz

Splen, v. Milz

Staining of osmium fixed Vestopal embedded tissue sections for light micros

copy 142

Stains, biological (7th ed.) (B)

188

Statistische Auswertung, v. Kerngrößenunterschiede

402

Index rerum

Stress, v. Reprints

Striatum, v. Corpus striatum

Submicroscopical structure, v. Electroniques, Elektronenmikroskopie, Transitional epithelium

Succinic dehydrogenase in the mammalian tongue with special reference to

gustatory epithelia 122

Sulfur, radioactive, v. Milz

Surrénales, glandes -, v. Nebenniere

Syrian hamster, v. Corpus striatum

Systematische Anatomie, v. Atlas (B)

Tageszyklische Untersuchungen an Rattenlebern (C)

$182(186)$

Teeth, v. Zähne

Telencephalon, v. Corpus striatum 
Teleost fish; studies of the acellular bone of teleost fish (II.: Response to fracture under normal and acalcemic conditions) Textur und Vascularisation der Fascien 46

Theorie physico-chimique et colloïdale de la caryocinèse (C) 171

Tilapia macrocephala, v. Teleost fish

Tissu adipeux; genèse et structure chez l'homme $\quad 232$

-; la lipogenèse au cours de la formation du tissu adipeux 316

Tissu osseux, fractures, v. Teleost fish

Tissue cultures, v. Bourgeons musculaires, Fibroblastes, Gonades embryonnaires, Mitochondries, Moelle osseuse, Myocardiques, Parabiose hétéro-

gène, Souris, Zellteilung Tongue, mammalian; succinic dehydrogenase in the mammalian tongue with

special reference to gustatory epithelia

Tooth, v. Zähne

Transitional epithelium of the rat urinary bladder in response to induced

physiological changes, histology, histochemistry and electron microscopy 297 Tritium, v. Milz

Übergangsepithel, v. Transitional epithelium

Ultrastructure, v. Electroniques, Elektronenmikroskopie, Transitional epithelium Unterkiefer, v. Acellular bone Urinary bladder of the rat, v. Transitional epithelium

Vaisseaux, v. Cerebral arteries, Cerebral infarction (B), Epiphysis, Vascular pattern,

Vascularisation

Vascular pattern of a growing and fullgrown human epiphysis

156

Vascularisation und Textur der Fascien

61

Vascularisation, v. Human epiphysis

Index rerum 403

Vergleichende Anatomie, v. Corpus striatum

Versammlungsberichte (C) $168 \mathrm{ff}$.

Vertebrata, v. Gehörorgan (B)

Vessels, v. Cerebral arteries, Cerebral infarction (B), Epiphysis, Vascular

pattern, Vascularisation Vessie (du rat), v. Transitional epithelium Vestopal-W; staining of

osmium fixed Vestopal embedded tissue sections for

light microscopy 142

Volume, poids sec et hydratation du noyau (C)

Volume du noyau, v. ADN, Zellkernvolumen

Wachstum, v. Human epiphysis

X-rays, v. Gonades embryonnaires, Pineal, Rayons X

Zähne; die Entwicklung der Innervation der menschlichen Zähne .... 322

Zellfunktion und Amitose, ihr Verhältnis (C) 175

Zellkerne, v. Divisions nucléaires

Zellkernvolumen und Mitoserate in der Zona glomerulosa und Zona fasci culate der Ratten-Nebenniere bei erhöhtem und erniedrigtem Koch-

salzangebot, Schwankungen (C) 180

Zellkulturen, v. Bourgeons musculaires, Fibroblastes, Gonades embryonnaires, Mitochondries, Moelle osseuse, Myocardiques, Parabiose hétéro-gène, Souris, Zellteilung Zellteilung; Wirkung des Heparins auf die Zellteilung und die Proliferationsprozesse (C) 185 
-; v. Amitose, Mitose, Divisions nucléaires, Elektronenmikroskopie

Zell..., v. Cell...

Ziege, v. Lemniscus

Zirbeldrüse, v. Pineal

Zunge, v. Succinic dehydrogenase

Book reviews - Livres nouveaux - Buchbesprechungen

Conn, H.J.: Biological stains (7th ed.) (The Williams and Wilkins Comp.,

Baltimore 1961) (Ref.: E. Reale) 188

Florey, E.: (Editor) Nervous inhibition (Proceedings of the second Friday

Harbor Symposium) (Pergamon Press, Oxford 1961)

(Ref.: H. G. Schwarzacher) 190

404

Index rerum

Graumann, W. und K. Neumann (Editores): Handbuch der Histochemie, Bd. 111,

Nucleoproteide: 2.Teil von: J. Bracket, M.S.Burstone, C. und R. Vendrely (Gustav Fischer,

Stuttgart 1959) (Ref.: R. Schenk) ... 295

Hofer, M., A.H.Schultz und D.Starck (Editores): Primatologia, Bd.II, Teil

2, 8.Lieferung: Basalganglien von: K.Feremutsch (Verlag S.Karger

Basel/New York 1961) (Ref.: E.Ludwig) 191

Hofer, M., A.H. Schultz und D. Starck (Editores): Primatologia, Bd. II, 5. Lie-

ferung: Das Ohr von: Cl. F. Werner und W. Lasinski) (Verlag S. Karger

Basel/New York 1960) (Ref.: E.Ludwig) 295

Huard, P. (Editeur): Leonard de Vinci, Dessins anatomiques (Dacosta, Paris

1961) (Ref.: A.Delmas) 293

Inke, G.: Präparation des Gehirns (Eine Anleitung zum makroskopischen

Studium) (G.Fischer, Jena 1961) (Ref.: E.Ludwig) 187

Komaromy, L.: Anatomische Gehirnsektion (Akadémiai Kiadó; Verlag der

Ungarischen Akademie der Wissenschaften, Budapest 1961) (Ref.:

E.Ludwig) $\quad 188$

Last, R.J.: Anatomy, regional and applied (second ed.) (J. \& A.Churchill

Ltd., London 1959) (Ref.: R.Schenk) 294

Wallraff, J.: Leitfaden der Histologie des Menschen (5.Aufl.) (Verlag Urban

und Schwarzenberg, München/Berlin 1962) (Ref.: 0. Bucher) .... 293

Werner, Cl. F,: Das Gehörorgan der Wirbeltiere und des Menschen (Georg

Thieme Verlag, Leipzig 1960) (Ref.: W. Platzer) 190

Wolf-Heidegger, $G_{2}$ : Atlas der systematischen Anatomie des Menschen Bd. 1

(2.Aufl.) (Verlag S.Karger, Basel/New York 1961) (Ref.: D.Starck) 189

Yates, P. O. and E. C. Hutchínson: Cerebral infarction: The role of stenosis

of the extracranial arteries (Her Majesty's Stationery Office, London

1961) (Ref.: S. Scheidegger) 189 